\title{
Synaptic Scaling Requires the GluR2 Subunit of the AMPA Receptor
}

\author{
Melanie A. Gainey, ${ }^{\star}$ Jennifer R. Hurvitz-Wolff, ${ }^{\star}$ Mary E. Lambo, and Gina G. Turrigiano \\ Department of Biology and Center for Behavioral Genomics, Brandeis University, Waltham, Massachusetts 02454
}

Two functionally distinct forms of synaptic plasticity, Hebbian long-term potentiation (LTP) and homeostatic synaptic scaling, are thought to cooperate to promote information storage and circuit refinement. Both arise through changes in the synaptic accumulation of AMPA receptors (AMPARs), but whether they use similar or distinct receptor-trafficking pathways is unknown. Here, we show that TTX-induced synaptic scaling in cultured visual cortical neurons leads to the insertion of GluR2-containing AMPARs at synapses. Similarly, visual deprivation with monocular TTX injections results in synaptic accumulation of GluR2-containing AMPARs. Unlike chemical LTP, synaptic scaling is blocked by a GluR2 C-tail peptide but not by a GluR1 C-tail peptide. Knockdown of endogenous GluR2 with an short hairpin RNA (shRNA) also blocks synaptic scaling but not chemical LTP. Scaling can be rescued with expression of exogenous GluR2 resistant to the shRNA, but a chimeric GluR2 subunit with the C-terminal domain swapped with the GluR1 C-terminal domain (GluR2/CT1) does not rescue synaptic scaling, indicating that regulatory sequences on the GluR2 C-tail are required for the accumulation of synaptic AMPARs during scaling. Together, our results suggest that synaptic scaling and LTP use different trafficking pathways, making these two forms of plasticity both functionally and molecularly distinct.

\section{Introduction}

Synaptic scaling and long-term potentiation (LTP) coexist at excitatory central synapses where they cooperate to maintain stability in neuronal output while selectively adjusting synaptic weights (Abbott and Nelson, 2000; Malenka and Bear, 2004; Turrigiano and Nelson, 2004). Some forms of LTP are induced by the regulated insertion of GluR1- and GluR2-containing heteromeric AMPA receptors (AMPARs) (Bredt and Nicoll, 2003; Shepherd and Huganir, 2007). The scaling up of synaptic strengths induced by prolonged treatment with TTX is also expressed by increased accumulation of GluR1 and GluR2 heteromeric AMPAR at spinal and neocortical synapses (O'Brien et al., 1998; Wierenga et al., 2005). GluR1 and GluR2 obey distinct trafficking rules in part because of differences in the sequences of their C-terminal domains (Shi et al., 2001), which differentially bind a large array of intracellular trafficking and scaffolding proteins (Malenka and Bear, 2004; Shepherd and Huganir, 2007). The subunit rules governing the expression of LTP at hippocampal CA1 synapses are relatively well defined: GluR1 is essential for LTP expression (Zamanillo et al., 1999), whereas GluR2 is not required (Jia et al., 1996). The role of the AMPAR subunits in synaptic scaling, however, remains relatively unexplored.

Two important functional distinctions between synaptic scal-

Received Aug. 8, 2008; revised March 6, 2009; accepted March 31, 2009.

This work was supported by National Institutes of Health Grant NS 36853 (G.G.T.). We thank Bryan Baxter and Kelly Tenney for technical assistance, Corinna Georgi for help designing RNAi constructs, Rob Tavares for help with immunostaining, Roberto Malinow for the original GluR2 construct and the GluR1 C-tail and GluR2 C-tail constructs, and Yasunori Hayashi for the original GluR2/1 C-tail and GluR1/2 C-tail chimera constructs.

${ }^{*} M$.A.G. and J.R.H.-W. contributed equally to this work.

Correspondence should be addressed to Gina G. Turrigiano, Department of Biology and Center for Behavioral Genomics, Brandeis University, Waltham, MA 02454. E-mail: turrigiano@brandeis.edu.

D01:10.1523/JNEUROSCI.3753-08.2009

Copyright $\odot 2009$ Society for Neuroscience $\quad$ 0270-6474/09/296479-11\$15.00/0 ing and LTP are their temporal and spatial scales. Some forms of LTP induce rapid insertion of AMPARs into the synaptic membrane on a time scale of minutes (Shi et al., 1999; Malenka and Bear, 2004), whereas synaptic scaling gradually and cumulatively increases synaptic AMPARs over a period of many hours (Turrigiano et al., 1998; Sutton et al., 2006; Ibata et al., 2008). Spatially, LTP can be induced in a synapse-specific manner in response to local synaptic activation (Matsuzaki et al., 2004), whereas synaptic scaling induced by TTX results in AMPAR accumulation at synaptic sites throughout the dendrite in response to a drop in postsynaptic firing (Ibata et al., 2008). These differences raise the possibility that LTP and synaptic scaling use distinct receptortrafficking pathways.

Here, we examine the roles of the GluR1, GluR2, and GluR3 subunits in the expression of synaptic scaling in rat visual cortical neurons. We show that synaptic scaling causes accumulation of GluR2-containing AMPARs in both cultured cortical neurons and in vivo. The GluR3 subunit poorly localizes to synaptic markers but shows increased surface accumulation during synaptic scaling, suggesting that GluR3 contributes to synaptic scaling but to a lesser extent than GluR1 or GluR2. A dominant-negative GluR1 C-tail was previously shown to block chemical LTP in our system (Watt et al., 2004). In this study, we show that the GluR1 $\mathrm{C}$-tail does not block the induction of synaptic scaling, whereas the GluR2 C-tail does. Knockdown of GluR2 (GluR2KD) using an short hairpin RNA (shRNA) also blocked the expression of synaptic scaling but had no effect on the induction of LTP. Furthermore, we show that in GluR2KD neurons, synaptic scaling can be rescued by expression of an exogenous GluR2 insensitive to the shRNA but not with a chimeric GluR2 in which the C-terminal domain has been swapped with the GluR1 C-terminal domain. Our results suggest that AMPAR accumulation during 
synaptic scaling and LTP are governed by distinct AMPAR trafficking rules and that interactions between the GluR2 C-tail and intracellular trafficking and scaffolding proteins are required for the accumulation of synaptic AMPARs during synaptic scaling.

\section{Materials and Methods}

Neuronal cultures, transfections, and immunostaining. Dissociated cultures were prepared from the visual cortex of postnatal day 2 (P2)-P4 Long-Evans rat pups and plated onto glass-bottomed dishes, as described previously (Pratt et al., 2003). All experiments were performed after 6-10 days in vitro (DIV). Cultures were transfected using lipofectamine reagent $48 \mathrm{~h}$ before recording or staining, unless otherwise noted. To induce synaptic scaling, neurons were treated with $1 \mu \mathrm{M}$ TTX for $24 \mathrm{~h}$, unless otherwise noted. Immunostaining using antibodies against postsynaptic density-95 (PSD-95; Affinity BioReagents; MA1045) and synapsin (Millipore Bioscience Research Reagents; AB1543) was performed as described previously (Rutherford et al., 1997). To visualize surface GluR1 (CalBioChem; PC246), GluR2 (Millipore Bioscience Research Reagents; MAB397), GluR3 (1:50; Millipore Bioscience Research Reagents; MAB5416), and green fluorescent protein (GFP; 1:100; Millipore Bioscience Research Reagents; AB3080P), live neurons were incubated with antibodies under nonpermeant conditions, as described previously (Wierenga et al., 2005). To ensure uniformity, data were acquired from dendrites branching off of the apical-like dendrite. All experimental conditions were run in parallel on sister cultures from the same dissociations; the mean intensity for experimental conditions was normalized to the mean intensity of control in sister cultures. The images in Figure 3, $A$ and $B$, and Figure $5 D$ were quantified with Openlab software (Improvision). All other images were quantified with MetaMorph software (Molecular Devices).

Culture electrophysiology. Whole-cell AMPA-mediated miniature EPSC (mEPSC) recordings were obtained and analyzed as described previously (Watt et al., 2000, 2004) at $25^{\circ} \mathrm{C}$, from a holding potential of -70 $\mathrm{mV}$ in the following artificial CSF (ACSF; in $\mathrm{mm}$ ): $126 \mathrm{NaCl}, 5.5 \mathrm{KCl}, 2$ $\mathrm{MgSO}_{4}, 1 \mathrm{NaH}_{2} \mathrm{PO}_{4}, 25 \mathrm{NaHCO}_{3}, 2 \mathrm{CaCl}_{2}, 14$ dextrose, $0.1 \mu \mathrm{M} \mathrm{TTX}, 0.02$ bicuculline, $0.025 \mathrm{D}$-APV. Chemical LTP was induced as described previously (Watt et al., 2004), and recordings were obtained from $15 \mathrm{~min}$ to $1.5 \mathrm{~h}$ after LTP induction. In a subset of experiments, neurons were treated for $1 \mathrm{~d}$ with the NMDA receptor (NMDAR) antagonist D-APV $(50 \mu \mathrm{M})$ or the metabotropic glutamate receptor-1 (mGluR1) antagonist LY $367385(30 \mu \mathrm{M})$ and the mGluR5 antagonist 2-methyl-6(phenylethynyl)-pyridine (MPEP) hydrochloride $(30 \mu \mathrm{M})$. Recordings with $V_{\mathrm{m}} \mathrm{s}>-54 \mathrm{mV}$, series resistances $\left(R_{\mathrm{s}}\right)>20 \mathrm{M} \Omega, R_{\text {in }}<150 \mathrm{M} \Omega$, or $<25$ mEPSCs were excluded. For the LTP experiments, recordings with $V_{\mathrm{m}} \mathrm{s}>-50 \mathrm{mV}$ were excluded. None of the transfection or drug conditions reported resulted in significant changes in $\mathrm{mEPSC}$ frequency, input resistance, or resting potentials. In addition, there were no significant changes in rise or decay times, with the exception of two conditions: GluR2KD plus RNAiI significantly slowed the decay time constant from 3.3 to 4.3 ms relative to untransfected neurons, and GluR2KD increased the rise time from 0.76 to 1.0 ms relative to untransfected neurons.

To measure rectification, spontaneous AMPA-mediated currents were measured at -60 and at $+50 \mathrm{mV}$, under conditions in which spontaneous AMPA currents could be isolated. For these experiments, the ACSF contained $0.01 \mu \mathrm{M}$ TTX (to raise spike threshold), APV (100 $\mu \mathrm{M})$, and MK801 $(50 \mu \mathrm{M})$ to block NMDA currents, and bicuculline $(20 \mu \mathrm{M})$ to block inhibitory potentials. The internal solution contained spermine $(120 \mu \mathrm{M})$ and QX314 (1 mM) to block spikes in the postsynaptic neuron and was Cs-based to facilitate voltage-clamping at $+50 \mathrm{mV}$. Under these conditions, well isolated spontaneous AMPA currents could be detected at both potentials; for each neuron, many events were detected and averaged at each potential. To compute the rectification index, the average peak amplitude at -60 was divided by the average peak amplitude at +50 ; the larger this value, the more rectification.

Visual deprivation and slice electrophysiology. Intraocular TTX injections were performed on P20-P23 rats 24 and $48 \mathrm{~h}$ before recording, as described previously (Desai et al., 2002; Maffei and Turrigiano, 2008). Coronal brain slices containing primary visual cortex were prepared from the control and deprived hemispheres, neurons were visualized with a $40 \times$ water-immersion objective using infrared-differential interference microscopy, and whole-cell recordings of AMPA-mediated mEPSCs were obtained from layer $2 / 3$ of monocular visual cortex; pyramidal neurons were identified based on morphology and post hoc reconstruction with biocytin fills, as described previously (Desai et al., 2002). Internal recording solution contained the following (in $\mathrm{mM}$ ): $20 \mathrm{KCl}, 100$ K-gluconate, 10 HEPES, 4 Mg-ATP, 0.3 Na-GTP, 10 phosphocreatine, and $0.2 \%$ biocytin. Neurons were voltage clamped to $-70 \mathrm{mV}$ in ACSF containing TTX $(0.2 \mu \mathrm{M})$, APV $(50 \mu \mathrm{M})$, and picrotoxin $(50 \mu \mathrm{M})$. Recordings were excluded if the $V_{\mathrm{m}} \mathrm{s}>-55 \mathrm{mV}$, the frequency $<0.1 \mathrm{~Hz}$, or the $R_{\mathrm{s}}>25 \mathrm{M} \Omega \mathrm{s}$. In a subset of experiments, the dicationic compound, IEM-1460 (100 $\mu \mathrm{M}$; Tocris Bioscience), was washed in for $10 \mathrm{~min}$ before recording.

\section{Construct design}

shRNA vector design. The mU6pro vector was used to express interfering shRNAs in neurons (Yu et al., 2002). The full mU6pro sequence, as well as protocols, can be found at http://sitemaker.umich.edu/dlturner.vectors. We used their protocol to insert hairpin sequences of our design into the vector but used 5' phosphorylated primers instead of unphosphorylated ones. Positive colonies were sequenced for accuracy. All primers were designed using Primer3 (http://frodo.wi.mit.edu/) with regions suitable for shRNA targeting chosen using Sfold (http://sfold.wadsworth.org/index.pl). Edited regions of GluR2 and the flip/flop alternative splice site were excluded from targeting. Before insertion into mU6pro, all shRNA sequences were screened with basic local alignment search tool, and any sequences with less than three base changes from other genes in mouse or rat were discarded. Of many hairpins screened, two gave good knockdown; we used constructs derived from the following primer set throughout the study: forward, 5' -/5Phos/TTT GTC CTC AGC ACT TTC GAT GGG AGC CAT CGA AAG TGC TGA GGA TTT TTC TAG AGC-3'; reverse, 5' -/5Phos/GGC CGC TCT AGA AAA ATC CTC AGC ACT TTC GAT GGC TCC CAT CGA AAG TGC TGA GGA-3'.

\section{RNAi-insensitive GluR2 design}

An RNAi-insenstive GluR2 (RNAiI) construct was designed using a GFP-GluR2 backbone from the Malinow lab (Hayashi et al., 2000). Sitedirected mutagenesis was used to change several wobble bases in the shRNA recognition site of the GFP-GluR2 so that complementarity to the hairpin was destroyed while maintaining the sequence of the expressed protein. Mutagenesis was performed using the following primers: forward, 5'-GGA TGG TGT CTC CGA TCG AGT CTG CAG AGG ATC TGT CTA AG-3'; reverse, 5' -CTT AGA CAG ATC CTC TGC AGA CTC GAT CGG AGA CAC CAT CC-3'. The GluR2(1-826)GluR1(823-889)-GFP (GluR2/CT1) and GluR1(1-822)-GluR2(827862)-GFP (GluR1/CT2) constructs were a gift from the Hayashi lab (Shi et al., 2001). The GluR2/CT1 chimera was made insensitive to the shRNA with the same primers used to create the RNAiI GluR2 construct. The GluR1/CT2 chimera is naturally insensitive to the GluR2 shRNA, since the shRNA does not target a sequence in the C-terminal domain. The GluR1(809-889)-GFP [GluR1 C-tail (GluR1CT)] and GluR2(813862)-GFP [GluR2 C-tail (GluR2CT)] constructs were gifts from the Malinow lab. All GluR2 constructs are the edited version of the subunit.

\section{Results}

Experiments were performed on cultured postnatal rat visual cortical neurons after 6-10 DIV or on acute rat visual cortical slices between P22-P24. Whole-cell recordings and fills were obtained from visually identified pyramidal neurons, as described previously (Desai et al., 2002; Maffei and Turrigiano, 2008). Cultures were transfected with the various constructs at low efficiency $48 \mathrm{~h}$ before recording or staining, unless otherwise noted. To visualize transfected neurons, all constructs were cotransfected with cyan fluorescent protein (CFP). Synaptic scaling was induced in cultured neurons by $1 \mathrm{~d}$ of TTX treatment $(1 \mu \mathrm{M})$, unless otherwise noted. For acute visual cortical slice experi- 
A
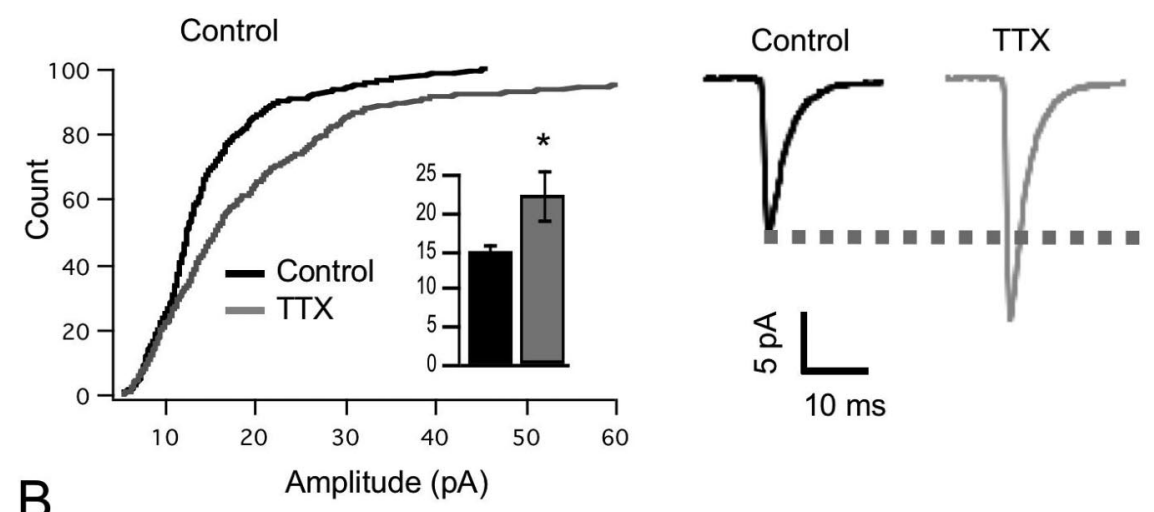

B

GluR1CT

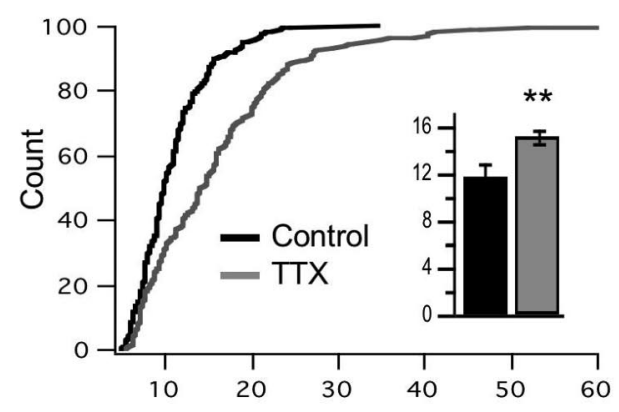

C

Amplitude (pA)

GluR2CT

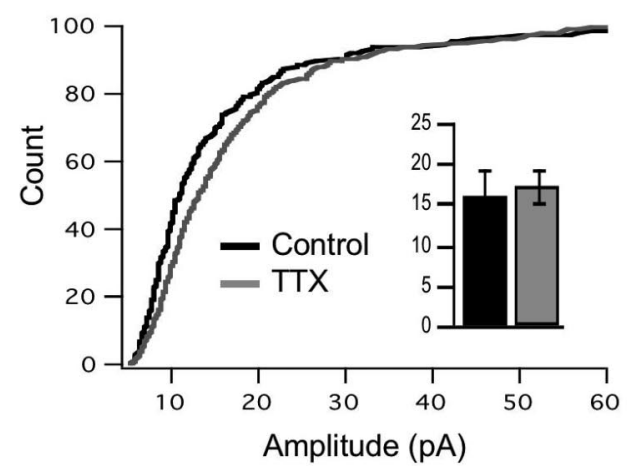

Figure 1. The GluR2 C-tail, but not the GluR1 C-tail, blocks synaptic scaling.A, Left, Cumulative histograms of mEPSC amplitude from control $(n=15)$ and TTX-treated ( $n=18)$ neurons (25 events per neuron). Inset, Average mEPSC amplitude for the same conditions. Right, Average mEPSC waveforms for the same conditions. $\boldsymbol{B}$, Left, Cumulative histograms of mEPSC amplitude for control ( $n=10)$ and TTX-treated ( $n=7)$ neurons transfected with the GluR1CT. Inset, Average mEPSC amplitude for the same conditions. Right, Average mEPSC waveforms for the same conditions. C, Left, Cumulative histograms of mEPSC amplitude for control $(n=9)$ and TTX-treated ( $n=14)$ neurons transfected with the GluR2CT. Inset, Average mEPSC amplitude for the same conditions. Right, Average mEPSC waveforms for the same conditions. All data here and below are reported as mean \pm SEM for the number of neurons indicated; Student's $t$ test, ${ }^{*} p<0.05,{ }^{* *} p<0.01$.

ments, synaptic scaling was induced with monocular TTX injections 24 and $48 \mathrm{~h}$ before recording, as described previously (Desai et al., 2002).

\section{The GluR2 C-tail, but not the GluR1 C-tail, blocks} synaptic scaling

Previous studies from our lab and others have shown that TTXinduced synaptic scaling is mediated by the accumulation of both GluR1 and GluR2 containing AMPAR in the postsynaptic membrane (O'Brien et al., 1998; Wierenga et al., 2005, 2006), but it is unknown whether domains particular to one or the other subunit

are necessary for the expression of synaptic scaling. We began with a dominantnegative approach; the GluR1 or GluR2 C-tail peptides were expressed in cultured neurons for $1 \mathrm{~d}$, and the cultures were treated with TTX for $\sim 20 \mathrm{~h}$ before recording miniature EPSCs (mEPSCs). In untransfected neurons, $1 \mathrm{~d}$ of TTX significantly increased mEPSC amplitude (Fig. 1A) (control, $n=15$; TTX, $n=18$; TTX was $149 \pm 23.8 \%$ of control, $p<0.05$ ). The GluR1CT blocks LTP in hippocampal slices (Shi et al., 2001) and chemical LTP in our culture system (Watt et al., 2004). In neurons transfected with the GluR1CT, TTX significantly increased mEPSC amplitude (Fig. $1 B$ ) (control GluR1CT, $n=$ 10; TTX GluR1CT, $n=7$; $p<0.005$ ), although the magnitude of synaptic scaling in GluR1CT neurons was significantly smaller than for control neurons (130 \pm $10.3 \%$ and $149 \pm 23.8 \%$, respectively; corrected $t$ test, $p<0.005)$, suggesting that protein interactions with the GluR1 C-tail influence scaling but are not required for its expression. In contrast, the GluR2CT completely blocked synaptic scaling (Fig. 1C) (control GluR2CT, $n=9$; TTX GluR2CT, $n=14$, TTX was $107 \pm 24.7 \%$ of control, $p=0.74$ ), suggesting that protein interactions with the GluR2 subunit are essential for the expression of synaptic scaling. The GluR2 C-tail is involved in some forms of long-term depression (LTD) induction (Xia et al., 2000; Kim et al., 2001; Chung et al., 2003; Steinberg et al., 2006), raising the possibility that scaling up of synaptic strengths during TTX treatment could be because of block of ongoing LTD in our cultures. To rule this out, we directly blocked NMDARdependent and mGluR-dependent LTD to see if this mimicked the effects of prolonged TTX treatment. Treatment for $1 \mathrm{~d}$ with the NMDAR antagonist D-APV (50 $\mu \mathrm{M})$, or the mGluR1 and mGluR5 antagonists LY $367385(30 \mu \mathrm{M})$ and MPEP hydrochloride $(30 \mu \mathrm{M})$, did not significantly increase quantal amplitude (both drugs slightly, but not significantly, decreased quantal amplitude: control: $17.7 \pm 1.6 \mathrm{pA}$, $n=12$; D-APV: $15.2 \pm 1.0 \mathrm{pA}, n=9$; LY 367385 and MPEP hydrochloride: $12.9 \pm 1.2 \mathrm{pA}, n=6$; ANOVA, $p=0.19)$, suggesting that TTX does not increase quantal amplitude by blocking ongoing LTD.

\section{Synaptic scaling causes accumulation of GluR2-containing AMPAR}

Synaptic GluR2 and GluR1 increase in parallel during synaptic scaling (Wierenga et al., 2005), suggesting that TTX-induced scaling leads to accumulation of GluR2-containing AMPAR. To examine the GluR2 content of functional synaptic AMPAR before and after synaptic scaling, we determined the rectification of 
AMPAR-mediated currents, since AMPAR without GluR2 are strongly rectifying, whereas those with GluR2 are not (Washburn et al., 1997). There was little rectification at synapses onto control neurons, indicating that most functional synaptic AMPARs contain GluR2 under control conditions. Furthermore, TTX treatment did not affect rectification, as expected if synaptic scaling increases the accumulation of GluR2-containing AMPAR (Fig. 2A).

To determine whether synaptic scaling in vivo also induces accumulation of GluR2-containing synaptic AMPARs, we induced synaptic scaling using $2 \mathrm{~d}$ of intraocular TTX into one eye starting at postnatal day 20 . We then cut acute visual cortical slices from the deprived and nondeprived hemispheres and recorded $\mathrm{mEP}$ SCs from layer $2 / 3$ pyramidal neurons in the monocular portion of primary visual cortex. As shown previously (Desai et al., 2002; Maffei and Turrigiano, 2008), 2 d of visual deprivation significantly increased mEPSC amplitude (Fig. 2B) (control, $n=$ 27; visual deprivation, $n=31$; visual deprivation significantly bigger than control, $p<0.02)$. In a subset of recordings, we washed in the dicationic compound IEM$1460(100 \mu \mathrm{M})$, which selectively blocks GluR2-lacking AMPAR (Magazanik et al., 1997; Buldakova et al., 2007). IEM-1460 induced a small but similar reduction in mEPSC amplitude in control and visually deprived neurons (Fig. 2C,D) (control, $96 \pm 6.1 \%$ compared with baseline, $n=$ 10 ; visual deprivation, $95 \pm 5 \%$ compared with baseline, $n=8$ ), indicating that only a small fraction of functional AMPAR lack GluR2 under both control and deprived conditions.

\section{GluR2 knockdown and compensation by other subunits}

LTP is preserved in GluR2 knock-out mice (Jia et al., 1996; Meng et al., 2003), but the role of GluR2 in synaptic scaling has not been assessed. To avoid possible compensatory mechanisms triggered by genetic deletion of GluR2, we used RNAi against GluR2 to acutely knock down expression in individual cultured cortical pyramidal neurons (GluR2KD). Expression of an shRNA directed against a sequence unique to the GluR2 subunit in individual pyramidal neurons reduced the length density of synaptic puncta expressing detectable levels of GluR2 to $\sim 20 \%$ of control after $24 \mathrm{~h}$ (Fig. $3 A, B)$ and reduced the intensity of staining at remaining puncta by $35 \%$. The length density of GluR1 and PSD-95 puncta was unaffected (Fig. $3 A, B$ ). There was little rectification at synapses onto control neurons. In contrast, synapses onto GluR2KD neurons showed pronounced rectification, indicating that GluR2 is greatly reduced or absent from synaptically localized AMPAR in these neurons (Fig. 3C,D). Despite the loss of GluR2 at synapses, mEPSCs were normal in both amplitude
B
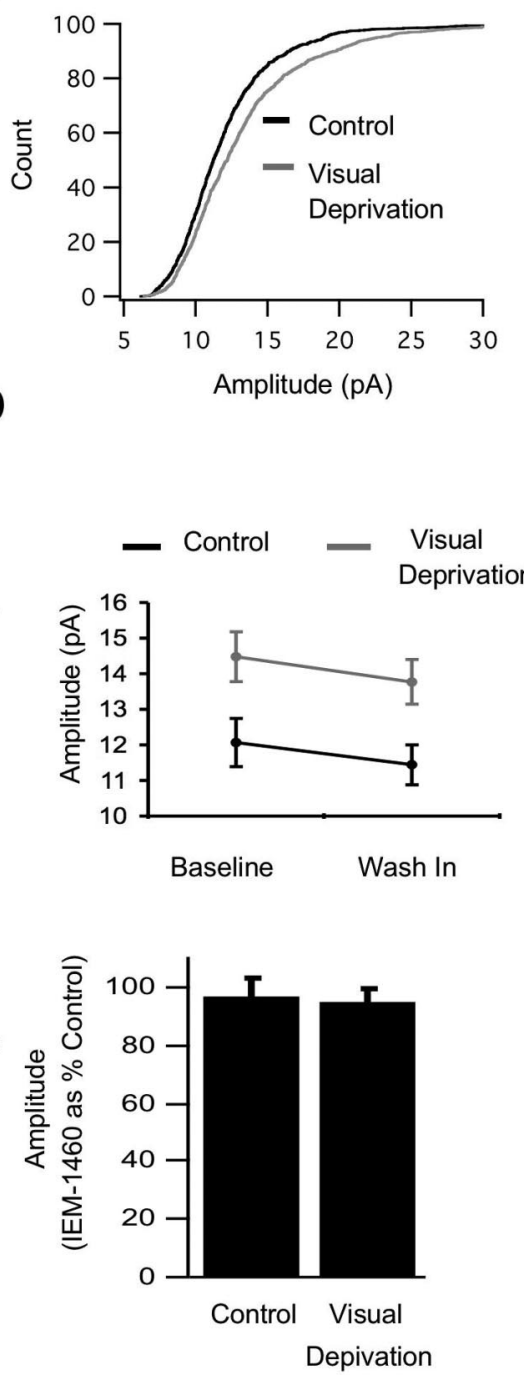

Figure 2. Synaptic scaling induces accumulation of GluR2-containing AMPARs both in vitro and in vivo. A, Left, Example AMPA-mediated currents at $-60 \mathrm{mV}$ and $+50 \mathrm{mV}$ for a TTX-treated neuron. Right, Rectification index for control $(n=9)$ and TTX $(n=6) . B$, Cumulative histogram of mEPSCs from layer $2 / 3$ pyramidal neurons in acute slices from monocular $V 1$, from the control hemisphere (control: $n=27,50$ events per neuron) and from the deprived hemisphere (visually deprived: $n=31,50$ events per -1460. Top, Neuron from the control hemisphere; bottom, neuron from the visually deprived hemisphere. D, Top, Average amplitude in IEM-1460 expressed as percentage of baseline in same condition \pm the propagated SEM for neurons from the control $(n=10)$ and visually deprived $(n=8)$ hemispheres.

and frequency (Fig. $3 E-G$ ). Baseline miniature transmission is thus unimpaired in neurons with acute GluR2 knockdown. Since baseline transmission was unperturbed in GluR2KD neurons, we wished to know which AMPAR subunits compensate for loss of GluR2. To examine the localization of the other major AMPAR subunits, GluR1 and GluR3, in control neurons, we performed immunohistochemistry under nonpermeant conditions and counterstained for the synaptic marker synapsin. GluR3 had a punctate distribution but a significantly lower colocalization rate with synapsin than GluR1 (Fig. $4 A, B$ ) (GluR1: $n=8$ neurons, 155 puncta; $51 \pm 6 \%$; GluR3: $n=8$ neurons, 141 puncta; $25 \pm$ $5 \%$; $p<0.005$ ), suggesting that GluR3 contributes to baseline transmission to a lesser extent than GluR1. To determine if GluR1 or GluR3 compensates for GluR2 loss, we quantified the intensity of surface GluR1 and GluR3 puncta in control and 
A

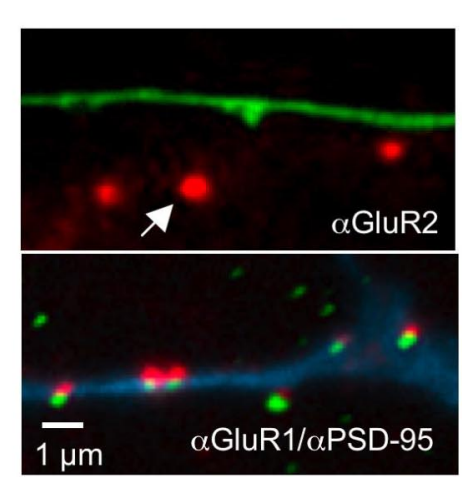

B
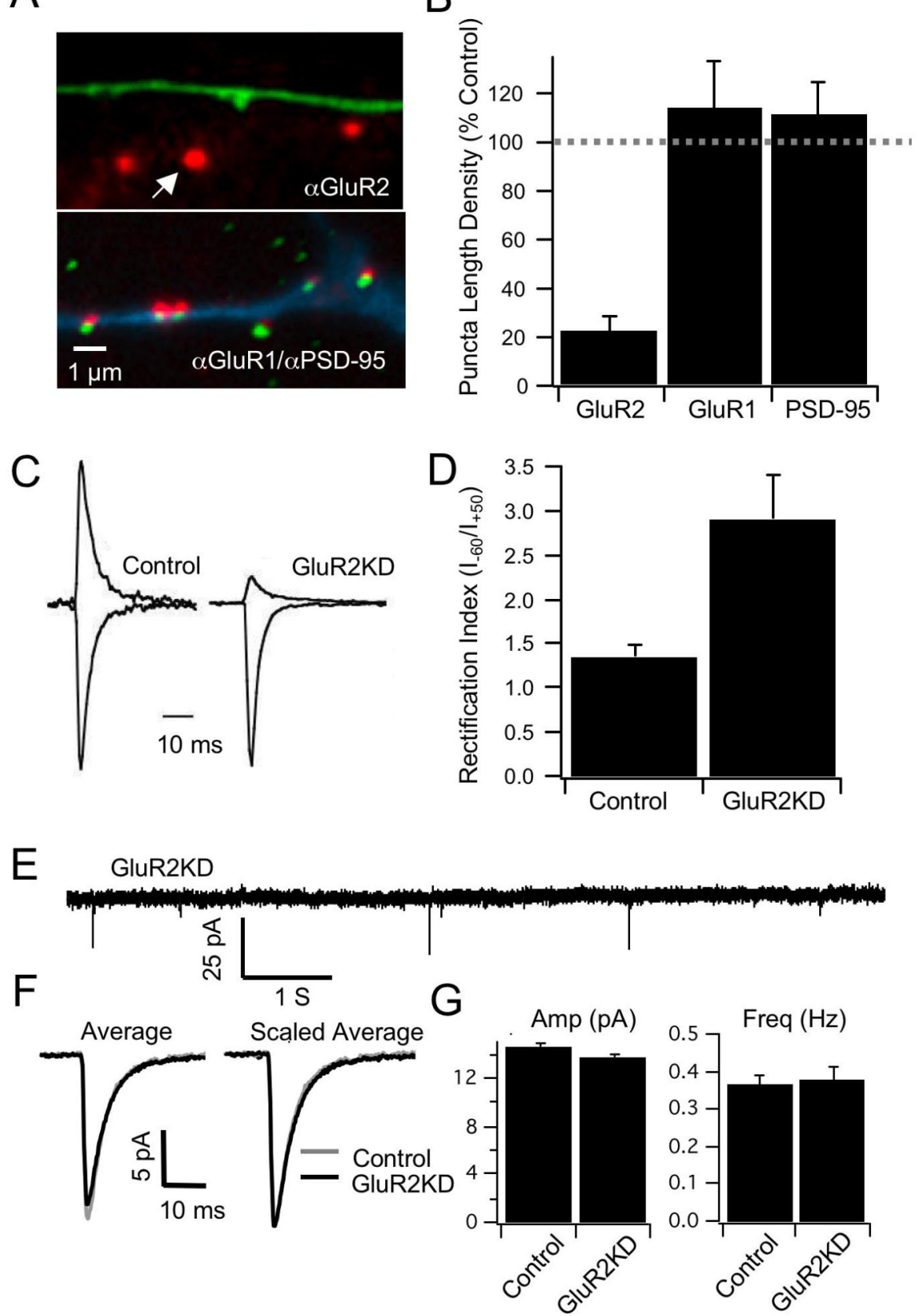

Figure 3. RNAi GluR2KD. A, Top, GluR2KD dendrite (green), $1 \mathrm{~d}$ after transfection with the shRNA against GluR2, labeled against endogenous GluR2 (red); note nearby untransfected dendrite with normal GluR2 labeling (arrow). Bottom, GluR2KD dendrite (blue) labeled against GluR1 (red) and PSD-95 (green). $\boldsymbol{B}$, Length density (puncta/micron) of GluR2, GluR1, and PSD-95 puncta in GluR2KD neurons compared with transfection with control construct ( $n=13,11$, and 9 for GluR2KD; 21, 14, and 11 for control). C, Example AMPA-mediated currents at $-60 \mathrm{mV}$ and $+50 \mathrm{mV}$ for control and GluR2KD neurons. $D$, Rectification index for control $(n=9)$ and GluR2KD $(n=8)$ neurons. $\boldsymbol{E}-\mathbf{G}, \mathrm{mEPSC}$ recordings from control and GluR2KD neurons $(n=10$ and 9$)$. *Different from control, $p<0.01$. All statistical tests here and below were ANOVAs as appropriate, followed by corrected two-tailed Student's $t$ tests unless noted otherwise.

GluR2KD neurons. The GluR1 puncta intensity significantly increased in GluR2KD neurons (Fig. 4C,D) (control: $n=6$ neurons, 284 puncta; GluR2KD: $n=6$ neurons, 337 puncta; GluR2KD was $169 \pm 23 \%$ of control; $p<0.05$ ). There was also a small, but not significant, increase in GluR3 puncta intensity (Fig. $4 C, D$ ) (control: $n=6$ neurons, 211 puncta; GluR2KD: $n=7$ neurons, 322 puncta; GluR2KD was $123 \pm 18 \%$ of control; $p=$ $0.24)$. We were unable to determine the colocalization of GluR2 and GluR3 since the N-terminal GluR2 and N-terminal GluR3 antibodies are from the same species. However, surface GluR1 showed little colocalization with GluR3 in control neurons, and there was no significant change in colocalization in GluR2KD neurons (Fig. $4 F$ ) (control: $n=11$ neurons, 737 puncta; $16 \pm 2 \%$; GluR2KD: $n=7$ neurons, 738 puncta; $18 \pm 4 \%$; $p=0.64)$. Together, these data suggest that compensation mainly occurs through an increase in GluR1 (possibly through formation of homomeric GluR1 receptors) with a smaller contribution from GluR3. Since the role of GluR3 in synaptic scaling is unknown, we stained for GluR3 under nonpermeant conditions in control and TTX-treated neurons. There was a significant increase in GluR3 intensity at puncta that were colocalized with GluR1 (Fig. 4E) (TTX was $181 \pm 45 \%$ of control; $p<0.05)$; there was also an increase in intensity at all puncta (colocalized and noncolocalized), although this increase was not significant (Fig. 4E) (control: $n=5$ neurons, 260 puncta; TTX: $n=10$ neurons, 871 puncta; TTX was $140 \pm 25 \%$ of control; $p=0.18$ ). Furthermore, TTX treatment significantly increased the colocalization of GluR1 to GluR3 (Fig. $4 F$ ) (control: $n=11$ neurons, 737 puncta; $16 \pm 2 \%$; TTX: $n=10,619$ puncta, $23 \pm 3 \%$; $p<0.05)$. The GluR3 subunit thus contributes to synaptic scaling, although to a smaller degree than GluR1 or GluR2.

\section{GluR2 knockdown blocks synaptic scaling but does not block LTP}

Next, we asked whether synaptic scaling could be induced in GluR2KD neurons. TTX increased mEPSC amplitude in untransfected control neurons (Fig. 5A-C) (control, $n=9$; TTX, $n=15$; TTX was $153 \pm 19.6 \%$ of control; $p<0.05$ ) but not in GluR2KD neurons from the same dish (control GluR2KD, $n=8$; TTX GluR2KD, $n=10$; TTX was $97 \pm 10.4 \%$ of control; $p=0.80$ ). In control neurons, $24 \mathrm{~h}$ of TTX significantly increased the peak intensity of synaptic GluR1 as reported previously (Wierenga et al., 2005) but not in GluR2KD neurons from sister cultures (Fig. 5D). To control for potential offtarget effects of the shRNA, we cotransfected neurons with the shRNA against GluR2 and an RNAi-insensitive GluR2 construct. Neurons in which endogenous GluR2 was replaced with RNAiI GluR2 (KD plus RNAiI) showed normal synaptic scaling (Figs. 5B, 7B).

An NMDAR-dependent form of LTP can be induced at these cortical synapses by increasing correlated firing under conditions where NMDAR activation is enhanced and like many forms of LTP is blocked by NMDAR antagonists and by transfection of neurons with the GluR1 C-tail (Watt et al., 2004). To examine the dependence of this form of chemical LTP on GluR2, we induced 
A

B

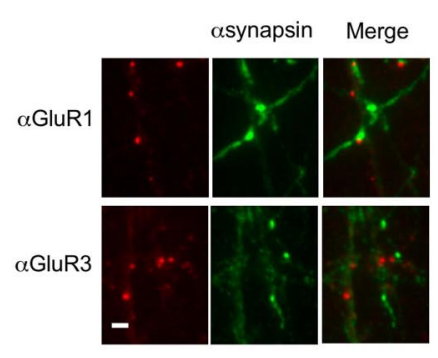

C

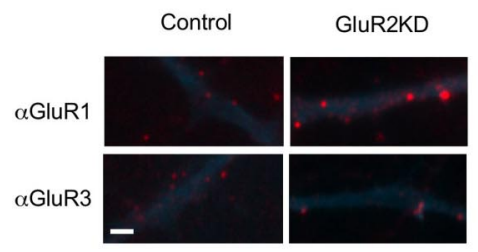

E

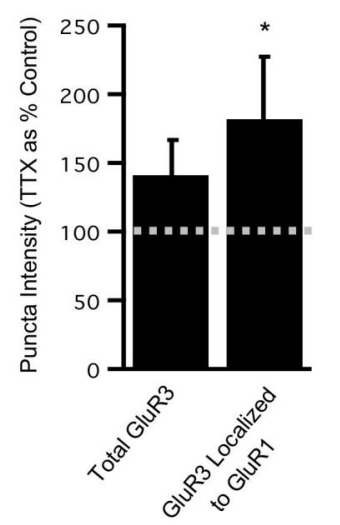

F

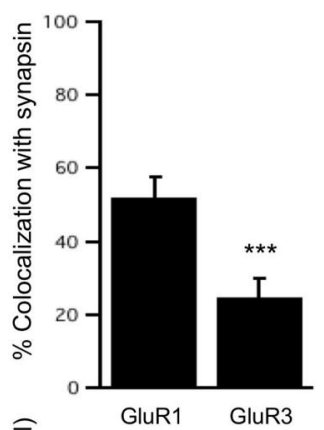

흔
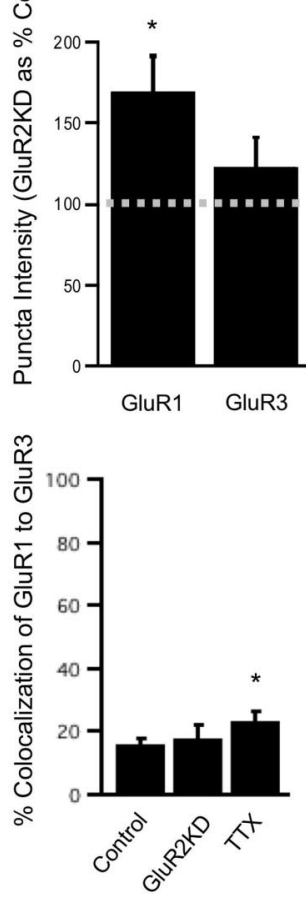

Figure 4. GluR1 compensates for GluR2 in GluR2KD neurons. $A$, Example of control dendrite labeled against endogenous surface GluR1 (top) or GluR3 (bottom) and synapsin; $\alpha$ GluR1 and $\alpha$ GluR3 in red, $\alpha$ synapsin in green. Scale bar, $2 \mu \mathrm{m}$. $\boldsymbol{B}$, Localization of GluR1 $(n=8)$ and GluR3 $(n=8)$ to synapsin. ${ }^{* *}$ GluR3 different from GluR1, $p<0.005$. C, Examples of control and GluR2KD dendrites labeled against endogenous surface GluR1 (top) or GluR3 (bottom); $\alpha$ GluR1 and $\alpha$ GluR3 in red, soluble CFP in blue. D, Total intensity of surface GluR1 and GluR3. GluR2KD is expressed as percentage of control for the same condition \pm the propagated SEM (GluR1, control: $n=6$; GluR2KD: $n=6$; GluR3, control: $n=6$; GluR2KD: $n=7$ ). *Different from control, $p<0.05$. $\boldsymbol{E}$, Total intensity of total surface GluR3 and GluR3 puncta localized to GluR1 puncta. TTX is expressed as percentage of control for the same condition \pm the propagated SEM (control, $n=5$; TTX, $n=10$ ). *Different from control, $p<0.05$. F, Localization of GluR1 to GluR3 in control $(n=11)$, GluR2KD $(n=7)$, and TTX $(n=10)$ neurons. *Different from control, $p<0.05$.

LTP in control and GluR2KD neurons (control, $n=18$; LTP, $n=$ 18 ; LTP was $149 \pm 15.8 \%$ of control; $p<0.001$ ). In contrast to the block of synaptic scaling, LTP was normal in GluR2KD neurons (Fig. $6 A, B$ ) (control GluR2KD, $n=12$; LTP GluR2KD, $n=11$; LTP was $153 \pm 25 \%$ of control; $p<0.05$ ). These data indicate that GluR2KD is not generally perturbing synaptic plasticity and that these two forms of synaptic plasticity have a differential dependence on GluR2.

One of the hallmarks of synaptic scaling is that the mEPSC amplitude distribution is scaled up proportionally by activity

blockade (Turrigiano et al., 1998; Desai et al., 2002). Like synaptic scaling during bath application of TTX, chemical LTP is induced in a network-wide manner that potentially affects all or most excitatory synapses, but whether chemical LTP produces proportional changes in mEPSC amplitude has not been examined. To compare synaptic scaling and chemical LTP, we rank ordered the mEPSC distributions by amplitude and plotted the amplitude before plasticity against the amplitude after plasticity (Fig. 6C). As described previously (Turrigiano et al., 1998), for synaptic scaling, this relationship was linear with a slope $>1$, and when scaled down multiplicatively by the parameters of the linear fit, the TTX distribution was almost perfectly superimposable on the control distribution (Fig. 6D). In contrast, the LTP data were poorly fit by a linear function, and when scaled down by the parameters of the linear fit, the LTP distribution was not superimposable on the control distribution (Fig. 6D). The relationship between control and LTP amplitudes was better captured by an exponential fit (Fig. 6C), suggesting that unlike synaptic scaling chemical LTP has a larger postsynaptic effect on strong synapses than on weak synapses.

\section{The GluR2 C-tail is required for the expression of} synaptic scaling

GluR2 contains several domains that are important for regulating its trafficking and synaptic accumulation (Malenka and Bear, 2004; Shepherd and Huganir, 2007). The C-terminal domain in particular has a number of sites that mediate interactions with scaffolding and trafficking proteins. Although several forms of LTD depend on these C-tail interactions, LTP does not (Malinow and Malenka, 2002; Malenka and Bear, 2004). To test the role of this domain in synaptic scaling, we modified the RNAiI GluR2 by swapping the GluR2 C-tail domain with the GluR1 C-tail domain to create a chimeric, RNAi-insensitive receptor (GluR2/CT1). Whereas replacement of endogenous GluR2 with the RNAiI GluR2 rescues synaptic scaling (Figs. 5B, $7 B$ ), replacement with the GluR2/CT1 (KD plus GluR2/CT1) results in normal basal transmission but blocks synaptic scaling (Fig. $7 A, B$ ) (control KD plus GluR2/CT1, $n=8$; TTX KD plus GluR2/CT1, $n=8$; TTX was $108 \pm 18.4 \%$ compared with control; $p=0.65)$. These data indicate that the GluR2 C-tail is essential for the regulated AMPAR accumulation that underlies synaptic scaling. We also tested the role of the GluR2 C-tail domain in scaling by creating the reverse chimera, GluR1 with a GluR2 C-tail domain (GluR1/ CT2). Replacement of endogenous GluR2 with the GluR1/CT2 chimera (KD plus GluR1/CT2) significantly increased baseline transmission (Fig. 7A,B) (control, $n=15$; control KD plus GluR1/CT2, $n=8$; control KD plus GluR1/CT2 was $151 \pm 23.8 \%$ compared with control; $p<0.01$ ) and occluded scaling (control KD plus GluR1/CT2, $n=8$; TTX KD plus GluR1/CT2, $n=8 ; p=$ 0.62). Expression of GluR1/CT2 without knockdown of endogenous GluR2 (GluR1/CT2) also increased baseline transmission, although not significantly (control, $n=15$; control GluR1/CT2, $n=7$; control GluR1/CT2 was $125 \pm 20.7 \%$ compared with control; $p=0.08$; data not shown). Control experiments where neurons were transfected with RNAiI GluR2 alone (RNAiI), or with the shRNA and RNAiI together, revealed that mEPSCs were of normal amplitude (Fig. 7A).

The GluR2 rescue constructs were tagged with GFP on the extracellular N-terminal domain. To verify that the chimeric AMPAR subunits traffic properly to the synaptic membrane, we used antibodies against GFP under nonpermeant conditions to detect surface receptors, followed by permeabilization and antibody localization of the synaptic marker PSD-95. The RNAiI 
A

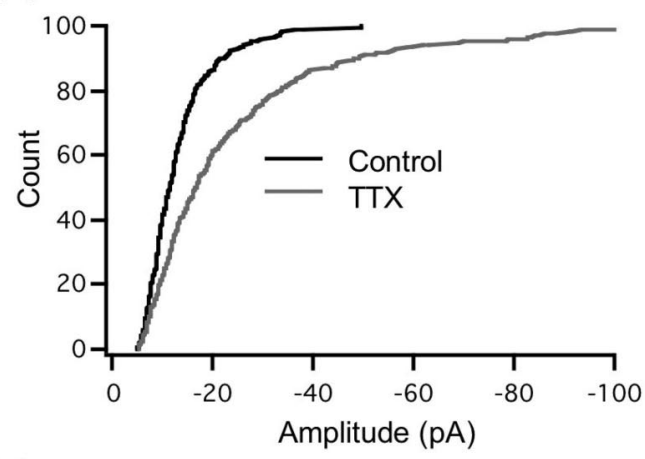

B

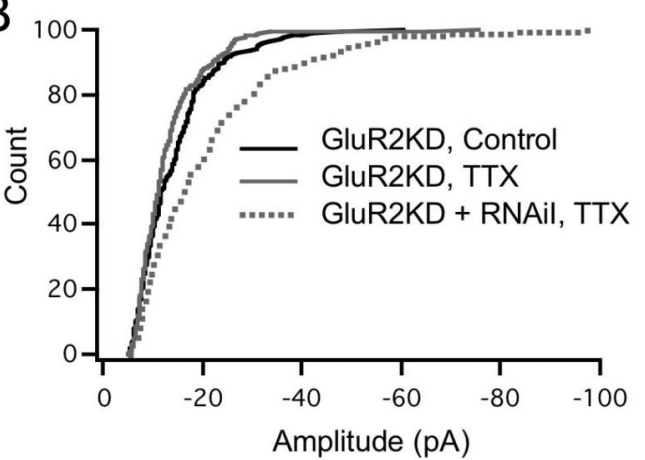

C

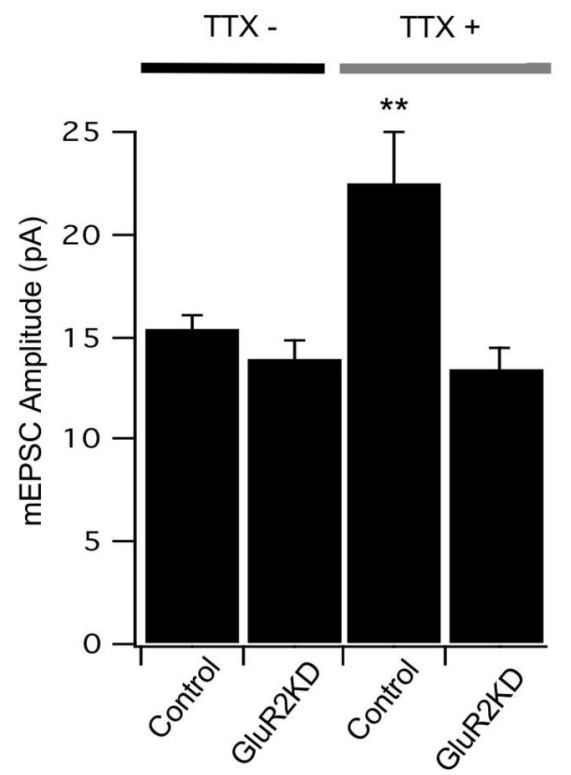

D

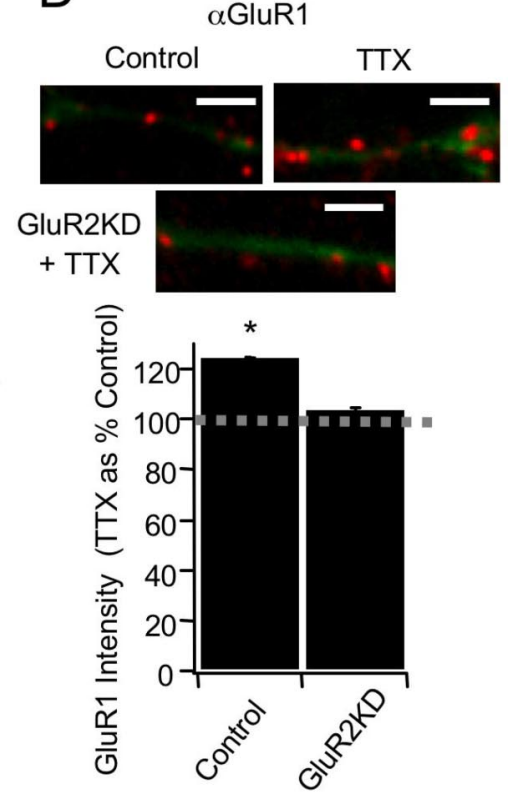

Figure 5. GluR2KD blocks synaptic scaling. $A$, Left, Cumulative histogram of $m$ EPSCs amplitude from untransfected neurons in control ( $n=14$ ) and TTX-treated ( $n=15$ ) conditions (25 events per neuron). Right, Average mEPSC waveforms for the same conditions. $\boldsymbol{B}$, Left, Cumulative histogram of mEPSC amplitude for control GluR2KD neurons $(n=8)$, TTX GluR2KD neurons $(n=$ $10)$, and TTX GluR2KD plus RNAil $(n=9)$ neurons. Right, Average mEPSC waveforms for the same conditions. C, Average mEPSC amplitude for indicated conditions, grown in control medium or $24 \mathrm{~h} \mathrm{TTX.} \mathrm{From} \mathrm{left} \mathrm{to} \mathrm{right,} n=14,8,15,10$. D, Top, Examples of staining against endogenous GluR1 from control and GluR2KD neurons after $24 \mathrm{~h}$ TTX treatment; $\alpha$ GluR1 in red, soluble GFP in green. Bottom, Peak intensity of GluR1 signal in synaptically localized puncta for neurons transfected with a control construct (control) or with the shRNA against GluR2 (GluR2KD); values from TTX-treated neurons expressed as percentage of untreated control $(n=11,15,15,17)$. Scale bar, $2 \mu \mathrm{m}$. *Different from control, $p<0.04$; ${ }^{* *} p<0.01$.

GluR2 on a GluR2 knock-down background showed a punctate distribution, with $53 \%$ colocalization of these surface puncta with PSD-95 (Fig. $7 A, B)(n=9$ neurons, 523 puncta). Similarly, $49 \%$ of GluR2/CT1 puncta on a GluR2 knock-down background colocalized with PSD-95 (Fig. 7C,D) $(n=$ 7 neurons, 672 puncta). It was not possible to compare this with colocalization rates for endogenous GluR2 because the N-terminal GluR2 and PSD-95 antibodies are from the same species; however, a previous study in our lab found that $55 \%$ of endogenous surface GluR1 puncta colocalize with PSD-95 (Wierenga et al., 2006). These chimeric subunits thus show comparable synaptic localization as endogenous AMPAR subunits, suggesting that the inability of the GluR2/CT1 chimera to rescue scaling is not attributable to a simple trafficking defect.

\section{Discussion}

Synaptic scaling is a form of homeostatic plasticity in which the strength of all of a neuron's synapses are proportionally scaled up or down in response to chronic changes in activity (Turrgiano et al., 1998). TTX-induced synaptic scaling proportionally increases GluR1 and GluR2 accumulation at synapses (Wierenga et al., 2005), but nothing is known about the underlying receptor-trafficking events. In principle, the GluR2 and GluR1 C-tails should selectively prevent GluR1dependent and GluR2-dependent receptor accumulation by binding to intracellular trafficking and scaffolding proteins known to interact with these C-terminal domains. Here, we show that the GluR1 C-tail does not block synaptic scaling, whereas the GluR2 C-tail does. To further probe the role of GluR2 in synaptic scaling, we used an shRNA to knock down GluR2 and found that this completely blocked synaptic scaling without affecting the induction of chemical LTP. Finally, we found that synaptic scaling can be rescued in GluR2KD neurons by coexpressing an RNAi-insensitive GluR2, but not an RNAi insensitive GluR2/CT1 chimera, demonstrating that regulatory sequences on the GluR2 C-tail are required for TTXinduced AMPAR accumulation. Together, our results provide evidence that GluR2 is essential for the expression of synaptic scaling and suggest that the regulated AMPAR accumulation underlying synaptic scaling and LTP use different receptortrafficking pathways.

The GluR1 and GluR2 subunits have three major protein-interaction domains: the extracellular $\mathrm{N}$ terminus, the poreforming region, and the intracellular $\mathrm{C}$ terminus (Hollmann and Heinemann, 1994; Song and Huganir, 2002). The differential trafficking of GluR1 and GluR2 has been attributed to different protein-binding sequences on the C-tail (Shi et al., 2001), although there is increasing evidence for the importance of 
the other domains in regulating AMPAR accumulation (Tomita et al., 2004; Nicoll et al., 2006; Panicker et al., 2008). The standard hippocampal model of AMPAR trafficking proposes that sequences on the GluR1 C-tail are required for regulated activity-dependent insertion, whereas sequences on the GluR2 C-tail are essential for constitutive, activity-independent recycling (Shi et al., 2001). This model has been complicated by the observations that AMPAR can be constitutively internalized and recycled in the absence of GluR2 (Biou et al., 2008). Furthermore, GluR2 lacking a C-tail, as well as chimeric GluR2 containing the GluR1 C-tail, can both traffic correctly to synapses in the absence of activity when introduced into GluR2 null neurons (Panicker et al., 2008). Finally, subunit trafficking rules are brain regiondependent. For example, LTD is unperturbed in the hippocampus of GluR2 knock-out animals (Meng et al., 2003) but blocked in cingulate cortex (Toyoda et al., 2007) and the cerebellum (Chung et al., 2003).

We found that acute knockdown of GluR2 does not impair baseline miniature synaptic transmission, as synapse density and mEPSC amplitude and frequency were very similar to control neurons. There are conflicting reports of whether chronic GluR2KO reduces baseline transmission; fEPSCs were unaffected in one study (Jia et al., 1996) but reduced in another (Meng et al., 2003). Some effects of chronic GluR2KO on baseline transmission could be attributable to developmental effects on the formation of local excitatory circuitry (Panicker et al., 2008). The published effects of GluR2 knockout on mEPSCs are variable; one study showed a modest but significant decrease in quantal amplitude in the GluR2 knock-out mouse (Panicker et al., 2008), whereas another reported that quantal amplitude was normal in the GluR2/GluR3 double knock-out mouse (Meng et al., 2003), consistent with our results using acute knockdown. Despite the difference in subunit composition of AMPAR in GluR2KD neurons, we observed no change in mEPSC kinetics, consistent with a study from hippocampal GluR2 knock-out mouse cultures (Panicker et al., 2008). This is likely because many factors in addition to subunit composition contribute to mEPSC kinetics, including editing and splice variants of subunits and the phosphorylation state of receptors (Angulo et al., 1997).

In GluR2KD neurons, baseline synaptic transmission was maintained by a compensatory increase in surface GluR1 but not GluR3. This is consistent with the mainly extrasynaptic localization of GluR3 in our neurons. The degree of colocalization between GluR1 and GluR3 remained low in GluR2KD neurons, suggesting that lack of GluR2 does not induce formation of
B

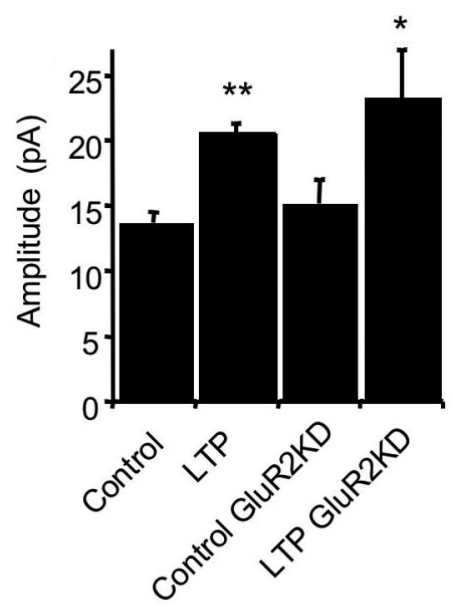

$\mathrm{D}$
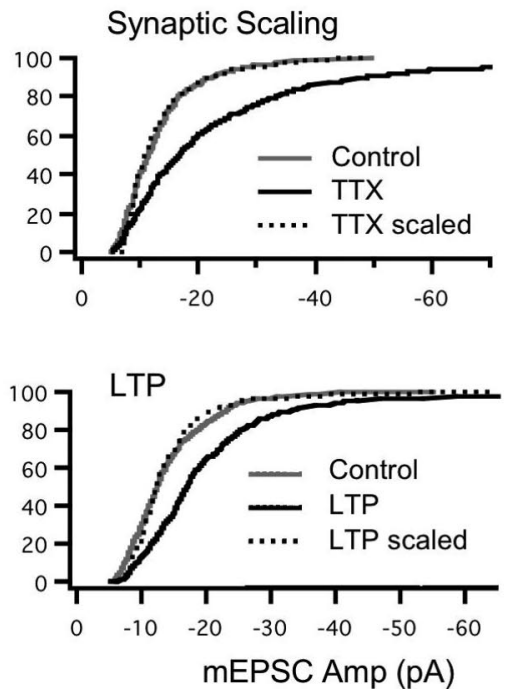

Figure 6. GluR2KD does not block chemical LTP. A, Average mEPSC waveforms for control and LTP conditions and LTP in GluR2KD neurons. $\boldsymbol{B}$, Average mEPSC amplitude for the indicated conditions, $n=19,12,18$, and, 11 respectively. ${ }^{*}$ GluR2KD LTP mEPSC amplitude by synaptic scaling but not by LTP. For both forms of plasticity, mEPSCs were rank ordered, and control values were plotted against potentiated values (either $1 \mathrm{~d}$ TTX treatment or chemical LTP protocol). The unity line (gray solid line) synaptic strength. For synaptic scaling (solid circles), the change in strength is well fit by a linear function with a slope $>1$ (gray for TTX are scaled down by the parameters of the linear fit in $(\boldsymbol{C})$, the distribution closely approximates the control distribution. Bottom, The same procedure for the LTP distribution produces a poor approximation of the control distribution.

GluR1/GluR3 heteromers, as has been shown in hippocampal neurons from a GluR2 knock-out mouse (Sans et al., 2003). It is surprising that GluR3, but not GluR2, is essentially extrasynaptic, since the two subunits have very similar C-tails and interact with many of the same trafficking proteins. One possible explanation is that GluR3 does not bind $\mathrm{N}$-ethylmaleimide-sensitive fusion protein, a trafficking protein thought to play an important role in targeting the insertion of GluR2 in the plasma membrane (Beretta et al., 2005). Although we found that GluR3 is mainly extrasynaptic, synaptic scaling increased the percentage of GluR1 puncta that were colocalized with GluR3 and also increased the intensity of GluR3 at puncta that were colocalized with GluR1. Together, these data suggest that GluR3 does contribute to synaptic scaling but to a lesser degree than GluR1 and GluR2. Al- 
A

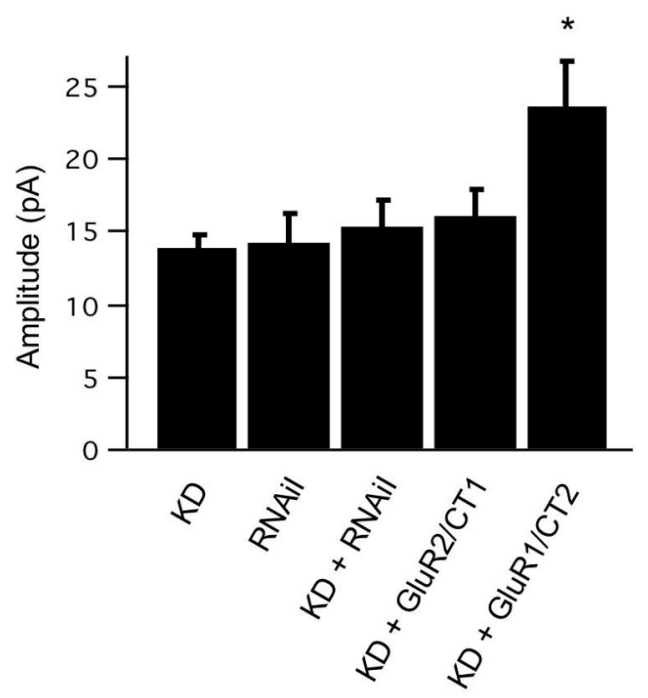

C
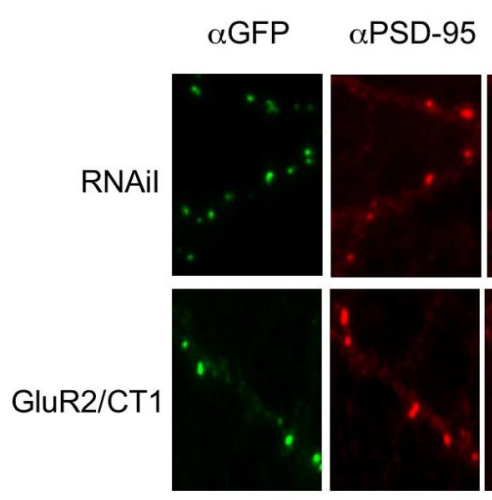

B

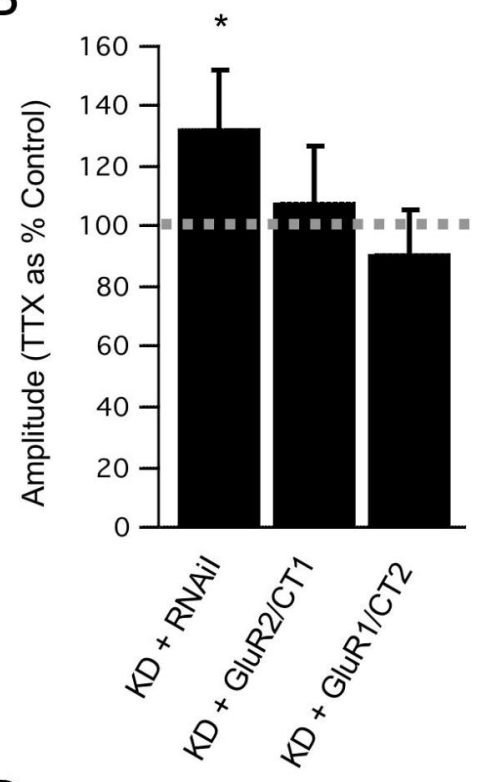

trafficking by increasing baseline calcium influx at synapses. Two results argue against this interpretation. First, chemical LTP is intact, arguing against a general calcium-induced defect in synaptic plasticity. Second, both intact GluR2 and the GluR2/CT1 chimera should decrease calcium permeability to a similar degree, yet the chimera fails to rescue scaling. Together, these data strongly suggest that synaptic scaling requires interactions with trafficking proteins such as ABP/GRIP (Osten et al., 2000) and PICK1 (Perez et al., 2001; Lu and Ziff, 2005) that are known to bind to the GluR2 C-tail. Whether this is because of a GluR2-dependent effect on receptor trafficking to the plasma membrane, tethering at synaptic sites, or internalization remains to be determined.

Interestingly, we also observed that a chimeric GluR1/C-tail 2 interferes with synaptic scaling. When introduced into GluR2 knock-down neurons, this construct increased synaptic transmission significantly, to a similar degree as $24 \mathrm{~h}$ of TTX treatment, and occluded the accumulation normally induced by TTX treatment. Since overexpressed intact GluR2 does not increase baseline transmission, this suggests either a change in the singlechannel conductance or the trafficking of the receptors. The trafficking could be altered if both C-tail and non-C-tail domain interactions are important in controlling baseline AMPAR accumulation and the GluR2 N terminus or transmembrane domain contains a sequence that normally puts a break on receptor accumulation. It is unclear at the moment how this construct interferes with synaptic scaling. One possibility is that synapses are "prescaled" because AMPAR have already occupied binding slots used during synaptic scaling. It is equally possible that synaptic scaling requires at least two trafficking steps for its expression, one

though it has been shown that GluR4 is developmentally regulated and has greatly reduced expression in cortical cultures by DIV 6-7 (Akaneya, 2007), it is possible that GluR4 also helps to maintain baseline transmission in GluR2KD neurons, a possibility we did not examine.

Contrary to the standard hippocampal model of receptortrafficking during LTP (Shi et al., 2001), our data suggests that GluR1/GluR2 heteromers can be trafficked via GluR2 interactions during synaptic scaling. GluR2 is essential for the regulated accumulation of AMPAR at the synapse during synaptic scaling. When introduced into GluR2 knock-down neurons, intact GluR2 can rescue synaptic scaling, whereas a GluR2/CT1 chimera cannot. This was not because of a simple trafficking defect, as both the intact and chimeric GluR2 trafficked correctly to the synaptic membrane. Another potential worry is that GluR2lacking AMPARs are calcium permeable (Isaac et al., 2007), raising the possibility that knocking down GluR2 disrupts receptor that relies on the GluR2 C-tail, and a second that relies on sequences in a non-C-tail domain.

Here, we show that synaptic scaling induces accumulation of functional GluR2-containing AMPARs both during TTXinduced scaling in culture and at layer $2 / 3$ synapses during visual deprivation-induced scaling in vivo. It has been suggested that synaptic scaling in layer $2 / 3$ induced by dark rearing results from accumulation of GluR2-lacking AMPARs, because dark rearing increases rectification at layer 4 to layer $2 / 3$ synapses (Goel et al., 2006). However, the effect of visual deprivation on layer 4 to $2 / 3$ synapses depends strongly on the mode of visual deprivation; for example, lid suture depresses excitatory transmission from layer 4 to $2 / 3$, whereas intraocular TTX enhances it (Maffei and Turrigiano, 2008), and it is not clear what effect brief dark rearing has on the absolute amplitude of transmission at this synapse. Here, we used mEPSC recordings, which sample from all the inputs onto layer 2/3 neurons, to assay the GluR2 content after synaptic 
scaling. We found that scaling up in response to monocular TTX injection results in increased accumulation of GluR2-containing AMPARs. These data are consistent with reports from cortical (Wierenga et al., 2005; Ibata et al., 2008), spinal (O'Brien et al., 1998), and hippocampal cultures (Sutton et al., 2006) that 1-2 d of activity blockade with TTX alone results in the insertion of GluR2-containing AMPARs. In contrast, several studies on homeostatic plasticity induced by chronic action potential and NMDAR blockade together have reported the selective accumulation of GluR1 homomers, with GluR2 playing little or no role (Ju et al., 2004; Thiagarajan et al., 2005; Sutton et al., 2006). Together with the results presented here, these studies suggest that blockade of spikes, or of spikes and NMDAR together, induce molecularly distinct forms of homeostatic plasticity that result from differential trafficking of GluR1 and GluR2. Synaptic scaling and many forms of LTP both result in increased AMPAR accumulation at synapses. Here, we show that a chemical form of LTP can still be induced in GluR2KD neurons that cannot express synaptic scaling. This indicates that AMPAR accumulation induced by synaptic scaling and LTP protocols result from different AMPAR trafficking rules with distinct subunit dependencies. A comparison of the mEPSC amplitude distribution after chemical LTP and synaptic scaling revealed different patterns of receptor insertion; although synaptic scaling scaled up synaptic strengths proportionally as described previously, chemical LTP did not, in keeping with the idea that these two forms of plasticity are both functionally and molecularly distinct. There is also much evidence for the role of GluR2 in the expression of some postsynaptic forms of LTD (Xia et al., 2000; Kim et al., 2001; Chung et al., 2003; Steinberg et al., 2006). The GluR2 C-tail interactions required for the expression of LTD are variable depending on the cell type and the induction method (Malinow and Malenka, 2002) and whether LTD and synaptic scaling result from reciprocal regulation of the same trafficking steps remains to be seen.

Together, our data strongly support the idea that increased AMPAR accumulation at neocortical synapses can be induced through two distinct pathways, an LTP pathway that depends on interactions with the GluR1 C-tail and a synaptic scaling pathway that depends on interactions with the GluR2 C-tail. This molecular distinction opens the possibility for designing manipulations that will selectively interfere with synaptic scaling while leaving LTP intact. These two forms of plasticity operate over different temporal and spatial scales, with LTP being rapid and local (Abbott and Nelson, 2000) and synaptic scaling being slower and global (Turrigiano and Nelson, 2004, Ibata et al., 2008). The differential dependence of LTP and synaptic scaling on AMPAR trafficking signals may facilitate the ability of neurons to engage in both complementary forms of plasticity without interference.

\section{References}

Abbott LF, Nelson SB (2000) Synaptic plasticity: taming the beast. Nat Neurosci 3:1178-1183.

Akaneya Y (2007) Activity regulates the expression of AMPA receptor subunit GluR4 in developing visual cortex. Eur J Neurosci 25:1641-1646.

Angulo MC, Lambolez B, Audinat E, Hestrin S, Rossier J (1997) Subunit composition, kinetic, and permeation properties of AMPA receptors in single neocortical nonpyramidal cells. J Neurosci 17:6685-6696.

Beretta F, Sala C, Saglietti L, Hirling H, Sheng M, Passafaro M (2005) NSF interaction is important for direct insertion of GluR2 at synaptic sites. Mol Cell Neurosci 28:650-660.

Biou V, Bhattacharyya S, Malenka RC (2008) Endocytosis and recycling of AMPA receptors lacking GluR2/3. Proc Natl Acad Sci USA 105:1038-1043.
Bredt DS, Nicoll RA (2003) AMPA receptor trafficking at excitatory synapses. Neuron 40:361-379.

Buldakova SL, Kim KK, Tikhonov DB, Magazanik LG (2007) Selective blockade of $\mathrm{Ca}^{2+}$ permeable AMPA receptors in CA1 area of rat hippocampus. Neuroscience 144:88-99.

Chung HJ, Steinberg JP, Huganir RL, Linden DJ (2003) Requirement of AMPA receptor GluR2 phosphorylation for cerebellar long-term depression. Science 300:1751-1755.

Desai NS, Cudmore RH, Nelson SB, Turrigiano GG (2002) Critical periods for experience-dependent synaptic scaling in visual cortex. Nat Neurosci 5:783-789.

Goel A, Jiang B, Xu LW, Song L, Kirkwood A, Lee HK (2006) Cross-modal regulation of synaptic AMPA receptors in primary sensory cortices by visual experience. Nat Neurosci 9:1001-1003.

Hayashi Y, Shi SH, Esteban JA, Piccini A, Poncer JC, Malinow R (2000) Driving AMPA receptors into synapses by LTP and CaMKII: requirement for GluR1 and PDZ domain interaction. Science 287:2262-2267.

Hollmann M, Heinemann S (1994) Cloned glutamate receptors. Annu Rev Neurosci 17:31-108.

Ibata K, Sun Q, Turrigiano GG (2008) Rapid synaptic scaling induced by changes in postsynaptic firing. Neuron 57:819-826.

Isaac JT, Ashby M, McBain CJ (2007) The role of the GluR2 subunit in AMPA receptor function and synaptic plasticity. Neuron 54:859-871.

Jia Z, Agopyan N, Miu P, Xiong Z, Henderson J, Gerlai R, Taverna FA, Velumian A, MacDonald J, Carlen P, Abramow-Newerly W, Roder J (1996) Enhanced LTP in mice deficient in the AMPA receptor GluR2. Neuron 17:945-956.

Ju W, Morishita W, Tsui J, Gaietta G, Deerinck TJ, Adams SR, Garner CC, Tsien RY, Ellisman MH, Malenka RC (2004) Activity-dependent regulation of dendritic synthesis and trafficking of AMPA receptors. Nat Neurosci 7:244-253.

Kim CH, Chung HJ, Lee HK, Huganir RL (2001) Interaction of the AMPA receptor subunit GluR2/3 with PDZ domains regulates hippocampal long-term depression. Proc Natl Acad Sci U S A 98:11725-11730.

$\mathrm{Lu}$ W, Ziff EB (2005) PICK1 interacts with ABP/GRIP to regulate AMPA receptor trafficking. Neuron 47:407-421.

Maffei A, Turrigiano GG (2008) Multiple modes of network homeostasis in visual cortical layer 2/3. J Neurosci 28:4377-4384.

Magazanik LG, Buldakova SL, Samoilova MV, Gmiro VE, Mellor IR, Usherwood PNR (1997) Block of open channels of recombinant AMPA receptors and native AMPA/kainate receptors by adamantine derivatives. J Physiol 505:655-663.

Malenka RC, Bear MF (2004) LTP and LTD: an embarrassment of riches. Neuron 44:5-21.

Malinow R, Malenka RC (2002) AMPA receptor trafficking and synaptic plasticity. Annu Rev Neurosci 25:103-126.

Matsuzaki M, Honkura N, Ellis-Davies GC, Kasai H (2004) Structural basis of long-term potentiation in single dendritic spines. Nature 429:761-766.

Meng Y, Zhang Y, Jia Z (2003) Synaptic transmission and plasticity in the absence of AMPA glutamate receptor GluR2 and GluR3. Neuron 39:163-176.

Nicoll RA, Tomita S, Bredt DS (2006) Auxiliary subunits assist AMPA-type glutamate receptors. Science 311:1253-1256.

O’Brien RJ, Kamboj S, Ehlers MD, Rosen KR, Fischbach GD, Huganir RL (1998) Activity-dependent modulation of synaptic AMPA receptor accumulation. Neuron 21:1067-1078.

Osten P, Khatri L, Perez JL, Köhr G, Giese G, Daly C, Schulz TW, Wensky A, Lee LM, Ziff EB (2000) Mutagenesis reveals a role for ABP/GRIP binding to GluR2 in synaptic surface accumulation of the AMPA receptor. Neuron 27:313-325.

Panicker S, Brown K, Nicoll RA (2008) Synaptic AMPA receptor subunit trafficking is independent of the C-terminus in the GluR2-lacking mouse. Proc Natl Acad Sci U S A 105:1032-1037.

Perez JL, Khatri L, Chang C, Srivastava S, Osten P, Ziff EB (2001) PICK1 targets activated protein kinase $\mathrm{C} \alpha$ to AMPA receptor clusters in spines of hippocampal neurons and reduces surface levels of the AMPA-type glutamate receptor subunit 2. J Neurosci 21:5417-5428.

Pratt KG, Watt AJ, Griffith LC, Nelson SB, Turrigiano GG (2003) Activitydependent remodeling of presynaptic inputs by postsynaptic expression of activated CaMKII. Neuron 39:269-281.

Rutherford LC, DeWan A, Lauer HM, Turrigiano GG (1997) Brain-derived 
neurotrophic factor mediates the activity-dependent regulation of inhibition in neocortical cultures. J Neurosci 17:4527-4535.

Sans N, Vissel B, Petralia RS, Wang YX, Chang K, Royle GA, Wang CY, O'Gorman S, Heinemann SF, Wenthold RJ (2003) Aberrant formation of glutamate receptor complexes in hippocampal neurons of mice lacking the GluR2 AMPA receptor subunit. J Neurosci 23:9367-9373.

Shepherd JD, Huganir RL (2007) The cell biology of synaptic plasticity: AMPA receptor trafficking. Annu Rev Cell Dev Biol 23:613-643.

Shi S, Hayashi Y, Esteban JA, Malinow R (2001) Subunit-specific rules governing AMPA receptor trafficking to synapses in hippocampal pyramidal neurons. Cell 105: 331-343.

Shi SH, Hayashi Y, Petralia RS, Zaman SH, Wenthold RJ, Svoboda K, Malinow R (1999) Rapid spine delivery and redistribution of AMPA receptors after synaptic NMDA receptor activation. Science 284: 1755-1757.

Song I, Huganir RL (2002) Regulation of AMPA receptors during synaptic plasticity. Trends Neurosci 25:578-588.

Steinberg JP, Takamiya K, Shen Y, Xia J, Rubio ME, Yu S, Jin W, Thomas GM, Linden DJ, Huganir RL (2006) Targeted in vivo mutations of the AMPA receptor subunit GluR2 and its interacting protein PICK1 eliminate cerebellar long-term depression. Neuron 49:845-860.

Sutton MA, Ito HT, Cressy P, KempfC, Woo JC, Schuman EM (2006) Miniature neurotransmission stabilizes synaptic function via tonic suppression of local dendritic protein synthesis. Cell 125:785-799.

Thiagarajan TC, Lindskog M, Tsien RW (2005) Adaptation to synaptic inactivity in hippocampal neurons. Neuron 47:725-737.

Tomita S, Fukata M, Nicoll RA, Bredt DS (2004) Dynamic interaction of stargazin-like TARPs with cycling AMPA receptors at synapses. Science 303:1508-1511

Toyoda H, Wu LJ, Zhao MG, Xu H, Jia Z, Zhuo M (2007) Long-term depression requires postsynaptic AMPA GluR2 receptor in adult mouse cingulate cortex. J Cell Physiol 211:336-343.
Turrigiano GG, Nelson SB (2004) Homeostatic plasticity in the developing nervous system. Nat Rev Neurosci 5:97-107.

Turrigiano GG, Leslie KR, Desai NS, Rutherford LC, Nelson SB (1998) Activity-dependent scaling of quantal amplitude in neocortical neurons. Nature 391:892-896.

Washburn MS, Numberger M, Zhang S, Dingledine R (1997) Differential dependence on GluR2 expression of three characteristic features of AMPA receptors. J Neurosci 17:9393-9406.

Watt AJ, van Rossum MC, MacLeod KM, Nelson SB, Turrigiano GG (2000) Activity coregulates quantal AMPA and NMDA currents at neocortical synapses. Neuron 26:659-670.

Watt AJ, Sjöström PJ, Häusser M, Nelson SB, Turrigiano GG (2004) A proportional but slower NMDA potentiation follows AMPA potentiation in LTP. Nat Neurosci 7:518-524.

Wierenga CJ, Ibata K, Turrigiano GG (2005) Postsynaptic expression of homeostatic plasticity at neocortical synapses. J Neurosci 25:2895-2905.

Wierenga CJ, Walsh MF, Turrigiano GG (2006) Temporal regulation of the expression locus of homeostatic plasticity. J Neurophysiol 96:2127-2133.

Xia J, Chung HJ, Wihler C, Huganir RL, Linden DJ (2000) Cerebellar longterm depression requires PKC-regulated interactions between GluR2/3 and PDZ-domain-containing proteins. Neuron 28:499-510.

Yu JY, DeRuiter SL, Turner DL (2002) RNA interference by expression of short-interfering RNAs and hairpin RNAs in mammalian cells. Proc Natl Acad Sci U S A 99:6047-6052.

Zamanillo D, Sprengel R, Hvalby Ø, Jensen V, Burnashev N, Rozov A, Kaiser KM, Köster HJ, Borchardt, Worley P, Lübke J, Frotscher M, Kelly PH, Sommer B, Anderson P, Seeburg PH, Sakmann B (1999) Importance of AMPA receptors for hippocampal synaptic plasticity but not for spatial learning. Science 284:1805-1811. 